This is the pre-peer reviewed version of the following article: Junhasavasdikul $\mathbf{D}$, et al. Cartoons on Facebook: a novel medical education tool. Med Educ. 2017 May;51(5):539-540. Epub 2017 Mar 14., which has been published in final form at doi: 10.1111/medu.13312. This article may be used for noncommercial purposes in accordance with Wiley Terms and Conditions for Self-Archiving.

\title{
Cartoons on Facebook: a novel medical education tool
}

Detajin Junhasavasdikul, MD,

Suthan Srisangkaew, MD,

Kanokporn Sukhato, DipMedEd, MD,

Alan Dellow, MMedEd, FRCGP.

What problem was addressed?

Cartoons, or comics, are being used increasingly in medical education. Publishing these on the internet can reduced publication costs and give access to an extremely wide audience. As the popularity of social media increases, these could also be effective platforms for cartoon-based medical learning. One study using cartoons on a website demonstrated an improvement in post-learning test scores of healthcare professionals ${ }^{1}$ but, to our knowledge, there are no reports of using graphic-novel cartoons on Facebook to teach a medical topic.

\section{What was tried?}

To evaluate the opinions of readers about this approach, a prospective trial was conducted; “Medical Academic Facilitation using Anthropomorphic Cartoon Illustrations (MAFAI): a pilot study". This was approved by the Ethics Committee of Ramathibodi Hospital, Mahidol University. We created a new non-animated comic-style cartoon aimed at teaching the physiology of breath sounds and their clinical examination. The story line used an anthropomorphic figure depicting a medical student questioning a resident about the physical examination of the lungs. The physiology was described using metaphors, diagrams and speech balloons, with the occasional use of humour for entertainment. The original version consisted of 3-4 panels (boxes) of cartoons per page with 29 pages in total. These were later rearranged into 49 pages to make them more readable.

The cartoon was posted on a Facebook webpage in March 2016. (Link: http://tiny.cc/MAFAl study) The final page encouraged readers to participate in an online survey during a 7-week period. Their personal characteristics, occupation and workplace were collected and a 5-point Likert-scale used to gather qualitative opinions of the cartoons.

\section{What lessons were learned?}

To date the cartoon has reached about 30,000 views, with 161 readers completed the questionnaire. Their mean age was $27.2( \pm 7.4)$ years and $60 \%$ were female. Most were doctors or medical students $(62.7 \%)$, followed by nurses and nursing students (19.3\%). All respondents were satisfied with our cartoon approach. More than $90 \%$ reported that the cartoons attracted their attention as well as helping them to memorize the content, understand the physiology, and improve their approach to conducting a thorough physical examination. The anthropomorphic characters were liked by $78 \%$ of the participants, only $10 \%$ suggesting that the cartoons would be improved by using human characters.

Our cartoon stayed "active" for a short period. A peak of about 10,000 views was reached on the day of publication, falling to 3,000 the following day and fewer than 100 views per day after a week. The prior popularity of the webpage probably played a role in determining these numbers, having 2,856 fans at baseline. The number of participants who thought that cartoon density per page was just right increased from $43 \%$ to $67 \%$ when the original 29 -page cartoon was replaced with the rearranged 49-page version, implying that less content density per page might be better.

Our study shows the popularity of using cartoon material on Facebook as a learning aid for a medical subject. Further research is needed to determine both the efficacy of this approach and the most preferable cartoon styles.

1. Sim MG, McEvoy AC, Wain TD, Khong EL. Improving health Professional's knowledge of hepatitis B using cartoon based learning tools: a retrospective analysis of pre and post tests. BMC medical education. 2014;14(1):244. 